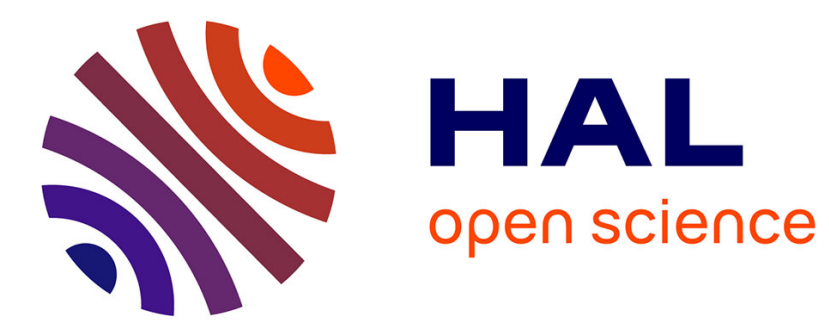

\title{
Category level object segmentation - learning to segment objects with latent aspect models
}

Diane Larlus, Frédéric Jurie

\section{To cite this version:}

Diane Larlus, Frédéric Jurie. Category level object segmentation - learning to segment objects with latent aspect models. VISAPP - 2nd International Conference on Computer Vision Theory and Applications, Mar 2007, Barcelona, Spain. pp.122-127. inria-00548681

\section{HAL Id: inria-00548681 \\ https://hal.inria.fr/inria-00548681}

Submitted on 20 Dec 2010

HAL is a multi-disciplinary open access archive for the deposit and dissemination of scientific research documents, whether they are published or not. The documents may come from teaching and research institutions in France or abroad, or from public or private research centers.
L'archive ouverte pluridisciplinaire HAL, est destinée au dépôt et à la diffusion de documents scientifiques de niveau recherche, publiés ou non, émanant des établissements d'enseignement et de recherche français ou étrangers, des laboratoires publics ou privés. 


\title{
CATEGORY LEVEL OBJECT SEGMENTATION Learning to segment objects with latent aspect models
}

\author{
Diane Larlus, Frédéric Jurie \\ LEAR Group, INPG-CNRS, INRIA Rhône-Alpes, France \\ firstname.name@inrialpes.fr
}

Keywords: Object segmentation, Latent aspect models.

\begin{abstract}
We propose a new method for learning to segment objects in images. This method is based on a latent variables model used for representing images and objects, inspired by the LDA model. Like the LDA model, our model is capable of automatically discovering which visual information comes from which object. We extend LDA by considering that images are made of multiple overlapping regions, treated as distinct documents, giving more chance to small objects to be discovered. This model is extremely well suited for assigning image patches to objects (even if they are small), and therefore for segmenting objects. We apply this method on objects belonging to categories with high intra-class variations and strong viewpoint changes.
\end{abstract}

\section{Introduction}

The problem of image segmentation and labeling image region is one of the key problems of computer vision. It consists in separating or grouping image pixels into consistent parts, brought to be elements that humans consider as individual objects or distinct object parts. This problem received a huge amount of attention in the past, and was originally addressed as an unsupervised problem. Many different methods have been developed, using various image properties such as color, texture, edges, motion, etc. (Haralick and Shapiro, 1985). It eventually turned out that image segmentation and image understanding were two closely related problems which cannot be solved independently. After being abandoned for a while, image segmentation came back into favor recently, taking advantage of recent advances of machine learning.

The goal addressed here is the segmentation of objects belonging to a given category ( the so-called figure-ground segmentation problem) assuming the category is defined by a set of training images. This is illustrated in Figure 1 for the "bicycle" category which is a very challenging category. The overall objective is to classify image pixels as being figure or ground. Objects can appear in any size and any position in the image. They can occur with widely varying appearances.
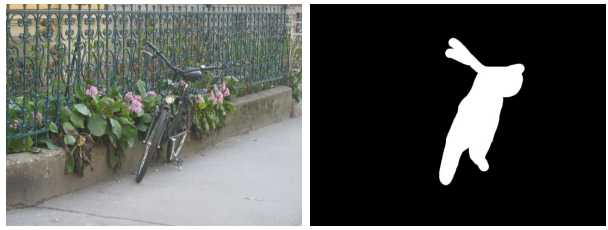

Figure 1: We show an image of the "bike" category, with its corresponding hand-made segmentation masks. Our goal is to design algorithms able to compute automatically this segmentation.

In such conditions, object segmentation is strongly linked to object detection and recognition. Indeed, segmenting objects requires learning object models from training images, as well as to search for occurrences of these models in images.

For this paper we will focus on difficult realcondition images where the objects can present extreme appearance variations (see Figure 1).

\subsection{Previous Work}

The method proposed in this paper is inspired by several related recent works, summarized below.

Leibe and Schiele (Leibe and Schiele, 2003) were among the first authors proposing to learn how to segment objects. Their method generates object hypothe- 
ses, without prior segmentation, that can be exploited to obtain a category-specific figure-ground segmentation. Training images are used to build a visual vocabulary of interest points, containing information about their relative positions as well as their corresponding segmentation masks.

Borenstein et al.(Borenstein et al., 2004) use the same idea of selecting informative patches from training images and then use their segmentation masks on new unseen images. They combine bottom-up and top-down approaches into a single process. The top-down approach uses object representation learned from examples to detect an object in a new image and provides an approximation to its segmentation. The bottom-up approach uses image-based criteria to define coherent groups of pixels that are likely to belong to the same part. The resulting combination benefits from both approaches.

Several approaches propose to use Conditional Random Field (CRF) for part-based detection (Quattoni et al., 2004) or segmentation (Kumar and Hebert, 2006). The previous authors extend the notion of CRFs to Discriminative Random Fields (DRFs) by exploiting probabilistic discriminative models instead of the generative models generally used with CRF.

Kumar et al. (Kumar et al., 2005) propose another methodology for combining top-down and bottom-up cues with CRFs. They combine CRFs and pictorial structures (PS). The PS provides good priors to CRFs for specific shapes and provides much better results.

None of the previous approaches is able to cope with occlusion. Win and Shotton (Winn and Shotton, 2006) were the first to address specifically this problem using an enhanced CRF. Their approach allows the relative layout (above/below/left/right) of parts to be modeled, as well as the propagation of long-range spatial constraints.

\subsection{Description of our approach}

Our approach shares many common features with the previously mentioned approaches. First, it combines bottom-up and top-down strategies.

The bottom-up process consists in sampling, and normalizing in size, dense image patches (small square image sub-windows), as in (Kumar and Hebert, 2006; Winn and Shotton, 2006), represented for subsequent processing by SIFT descriptors (Lowe, 2004). These descriptors are then vector quantized into a discrete set of labels so called visual words. Each patch is described by the word of the nearest centroid. This process is illustrated Figure 2. From this stage, images are seen as sets of visual words occurrences. As the process assigns figure/ground la-

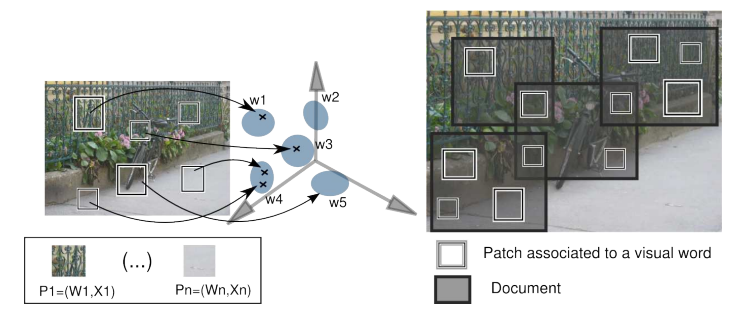

Figure 2: The visual vocabulary is obtained by vector quantizing a set of image patches descriptors.Images are modeled as sets of overlapping documents, each document being a set of patches.

bels to patches, the pixel level segmentation requires an additional process, responsible for combining labels carried by patches into pixel hypotheses.

The top-down process embeds object models and uses them to obtain a global coherence, by combining local information provided by the bottom-up process. Most of the models previously used in this context cannot be used here, because of the strong variation of object's appearance. Geometric models such as the Pictorial Structure (Kumar et al., 2005) or the Implicit Shape Model (Leibe and Schiele, 2003) would require a huge number of training images in order to capture the large variability of appearance. Approaches based on characteristic edge patches (Borenstein et al., 2004) are only usable when object outlines are sufficiently stable. As a consequence, it appears that a more flexible model is required to address such object categories.

For the recognition of complex object categories, the bag-of-words model (Csurka et al., 2004) has been shown to be one of the most effective. It was inspired from text classification; the text framework becomes applicable for documents corresponding to images, once images have been transformed into sets of visual words. More recently, techniques based on latent aspects were developed on top of this unordered word based representation and were applied first for text classification (Griffiths and Steyvers, 2004), and then for image classification (Sivic et al., 2005). Such models are usually coming from the probabilist Latent Semantic Analysis (pLSA) (Hofmann, 2001) or its Bayesian form, the Latent Dirichlet Allocation (LDA) (Blei et al., 2002). Visual words are considered as generated from latent aspect (or topics) and images are combination of specific distribution of topics.

Using this latent aspect based framework for segmenting images is appealing for several reasons. First because object appearances (topics) can be automatically discovered and learned, limiting the amount of supervision required. Second, the flexibility of such 


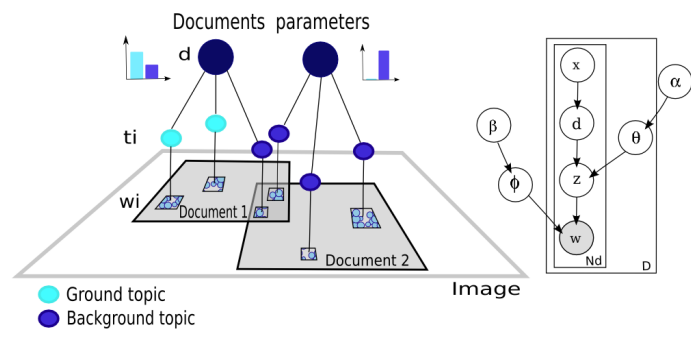

Figure 3: Left: 2 documents overlapping in an image. 2 of the patches seen as their visual words $(w)$ belong to the object topic $(t)$ and 4 of them belong to the background. The document distribution over topics is represented by the histograms. Right: the corresponding graphical model.

a framework can handle large variations in appearance and shape. However, objects have to cover large parts of images so they constitute dominant image topics. This is not the case when objects are small as in Figure 1. Furthermore, as no global geometric constraints are used, the proposed models are not well suited for the detection or segmentation tasks.

The main contribution of this paper is to propose a new graphical model for representing images and objects, addressing the two limitations previously raised. Our model, illustrated Figures 2 and 3, consists in describing images by a set of overlapping multi-scaled local documents. In this case, even small objects constitute the main topics of at least few documents and therefore can be discovered. Each image patch (visual word) belongs to several overlapping documents. The process of assigning labels (figure/ground labels) to patches is done at the document level, which is a semi-global level. However as documents are overlapping and share image patches, semi-local decisions are propagated all over the image, as MRFs do.

A training stage with some object examples is used to compute the priors which are then utilized in the model estimation of unseen images. Consequently, our model is semi-supervised.

The organization of the paper is as follows: in section 2 the proposed model and its estimation are described. The last section presents some experiments carried out on challenging natural scene images.

\section{Multi-document model}

\subsection{Description}

Images are considered as unordered sets of patches (rectangular small sub-images). Each patch is represented by a visual word together with its position in the image (see Figure 2). Our approach is generative: visual words are supposed to come from underlying factors denoted topics which describe latent aspects of images, as with the LDA model (Blei et al., 2002). In practice, we will use only two topics, one for representing background, the other for the object.

An image is described by a multitude of different documents $(d \in D)$ corresponding to overlapping regions of this image (see Figure 2). Positions and scales of documents are chosen to cover the image uniformly. Each document has its own distribution over the topics, denoted $\theta_{d}$. In contrast, within all documents, the probability for topics to generate visuals words is the same. Topic distribution over words, denoted $\phi$, is sampled from a Dirichlet distribution of hyper-parameter $\beta$. The model is illustrated Figure 3.

Modeling an image $I$ assumes that it is built according to the following generative process:

1. First, the distribution $\theta_{d} \sim \operatorname{Dir}(\alpha)$ is sampled for each document $d$, where $\operatorname{Dir}(\alpha)$ is a Dirichlet distribution of hyper-parameter $\alpha$, providing distributions over the latent topic factors,

2. For each observation (i.e., a patch associated to a visual word $w$ and a location $x$ ) :

(a) Equiprobably choose a document $d$ from the set of documents containing $x . P(d \mid x)=O$ if $x \notin d$ and $P(d \mid x)=\frac{1}{N}$ if $x \in d$, where $N$ is the total number of documents containing $x$.

(b) Draw a topic $z$ from the multinomial distribution of parameter $\theta_{d}: z \sim \operatorname{Mult}\left(\theta_{d}\right)$

(c) Finally draw a word $w$ conditional on $z$ from the multinomial distribution $\phi: w \sim \operatorname{Mult}(\phi)$.

The joint probability $P(w, d, z, x)$ is assumed to have the form of the graphical model shown in Figure 3. Marginalizing over topics $z$ and document $d$ determines the conditional probability $P(w \mid x, \alpha, \beta, I)$ :

$P(w \mid x, \alpha, \beta, I)=\sum_{d \in D} \int_{\theta} \sum_{z \in Z} P(w \mid z, \phi) P(z \mid d, \theta) P(d \mid x) d \theta$

where $w$ stands for the visual word, $x$ its position, $Z$ the set of latent topics, $D$ the set of documents, $I$ the image and $\theta$ and $\phi$ are the previously mentioned multinomial distributions.

\subsection{Model estimation}

In the previously described model, the patch position $x$ and its corresponding visual words $w$ can be directly observed. Hyper-parameters $\alpha$ and $\beta$ have fixed values that will be described later in this section. Estimating the model consists in computing the multino- 
mial distributions $\theta$ and $\phi$ with respect to their Dirichlet prior $\alpha$ and $\beta$ and knowing a set of $x$ and $w$ observed in images.

The estimation is done according to the maximum likelihood criterion: We collect $N$ images and observe the set of patches $\left(x_{1}, w_{1}\right), \ldots,\left(x_{N}, w_{N}\right)$. We want to compute $\theta$ and $\phi$ maximizing $P\left(\left(x_{1}, w_{1}\right), \ldots,\left(x_{N}, w_{N}\right) \mid \theta, \phi, \alpha, \beta\right)$.

Since the integral of the observation generation probability (equation 1) makes the direct optimization of the likelihood intractable, we estimate variables of interest by an approximate iterative technique called Gibbs sampling.

During this process we estimate topic affectations (hidden variables of the model) jointly with $\theta$ and $\phi$. $\theta$ and $\phi$ are never explicitly estimated, but instead the posterior distribution over the assignments of words to topics $P(z \mid w)$ is considered. This process has been proposed by (Griffiths and Steyvers, 2004) for the LDA model. Gibbs sampling works as follows: documents are initialized with equiprobable distribution over topics, then we iterate to estimate the posterior distribution $P(z \mid w)$.

In practice, the model includes only two topics, one for describing foreground patches, the other for background patches. However in a totally unsupervised framework, as we presented so far, foreground objects might not be automatically chosen as a topic of the model. The multi-documents model might capture other more frequent aspects of the image.

This is the reason why some extra supervision is added during the learning stage. A set of training images with possibly different levels of supervision is used to estimate foreground topic distribution over words. This is done with a standard LDA model (Blei et al., 2002) which is able to capture efficient topic distributions even with small supervision. This learned distribution is then used as a prior on the $\phi$ distribution for the test images.

It should be noted that for making the estimation possible we only process one test image at a time. We typically have thousands of documents per image. Processing all these images simultaneously would be infeasible. As a consequence, documents of different images become independent.

The hyper-parameters $\alpha$ and $\beta$ play an important role as they allow to control topics and visual words distribution. For $\alpha$, a small scalar value has been taken as in (Griffiths and Steyvers, 2004) in order to produce sparse and therefore specialized topics distribution. For $\beta$ the knowledge acquired during the training stage has been used. We used values proportional to the number of patches affected to topics and words. This prior has large values as the knowledge acquired by LDA model is strong, but during the estimation the $\phi$ distribution can still be adapted to a particular test image.

\subsection{From patches to segmentation}

At the end of the estimation process, all patches have a probability of being generated by one of the foreground/background topics. These patches correspond to the squared sub-window's pixels used to build visual words. To compute the probability for a pixel $p$ belonging to an object class (corresponding to topic $z$ ), we accumulate the knowledge on patches $\mathcal{P}$ containing the pixel. This is modeled by a mixture model, where weights (probability of a pixel to have been generated by a patch $P(p \mid \mathcal{P})$ ) are functions of the distance between the pixel and the center of the patch. We have $P(\operatorname{class}(p)=z) \propto \sum_{\mathcal{P}_{i} \ni p} P\left(t_{i}=z\right) P\left(p \mid \mathcal{P}_{i}\right)$ where $t_{i}$ stands for the topic of patch $\mathscr{P}_{i}$.

This can be seen as a summary of all labels provided for the same pixel. In regions where neighboring patches disagree, the confidence will be low; in contrast if neighboring patches agree, the probability for the pixel to belong to the object becomes higher.

\section{Experiments}

We tried to evaluate the soundness of our method by comparing segmentation it produces with hand ground truth segmentation. We chose to use the bike class of the Graz-02 datase ${ }^{1}$ because of its high complexity. It contains images with high intra-class variability on highly cluttered backgrounds. The ground truth is available for 300 images. It is given in terms of pixel segmentation masks (one example is shown Figure 1). These masks will be used to evaluate the quality of our segmentation.

Methodology We have shown in section 2.3 that our algorithm computes the probability for each image's pixel to belong to an object of a given category. On the other hand, we know ground truth pixels labels, given by the provided segmentation masks. It is therefore natural to evaluate the performance by computing a ROC curve for each image. The ROC curve represent the true positive rate $(T P)$ against the false positive rate $(F P)$, i.e., the rate of correct classification for the category of interest against the rate of object pixels misclassified. The true positive rate at equal error rate (EER) is the true positive rate at the curve point where $T P=1-F P$.

\footnotetext{
${ }_{1}^{1}$ available at http://www.emt.tugraz.at/pinz/data/GRAZ_02/
} 

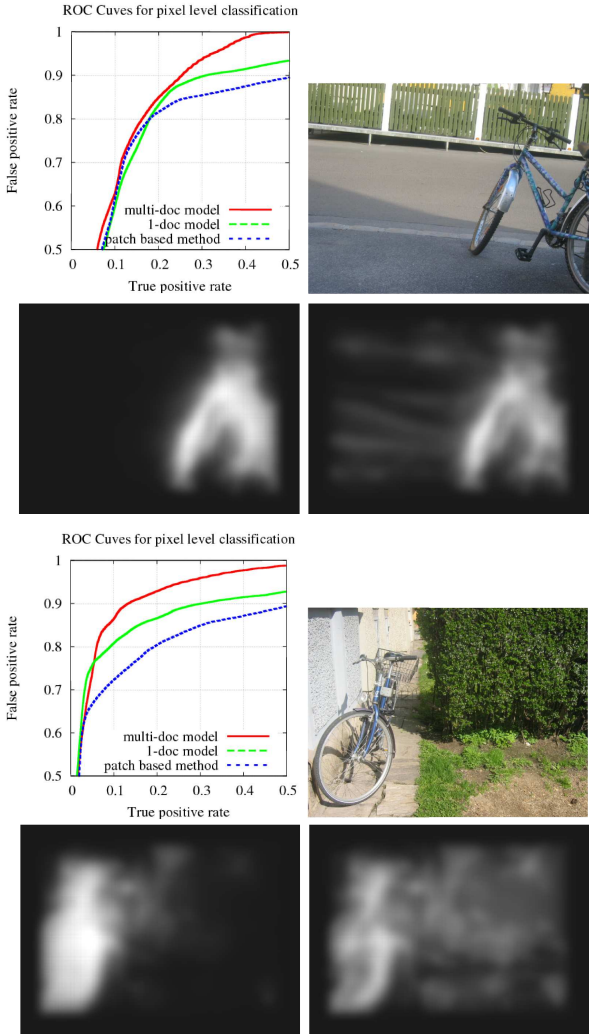

Figure 4: Comparison of our method with 2 baseline methods. For each original image (upper-right), the ROC curve for the 3 methods (upper-left), the probability map obtained by our method (bottom-left) and the one obtained with the single document method (bottom-right)

Experimental settings Patches are chosen at different scales according to a dense grid. This sampling is dense enough for all pixels to belong to several patches. These patches are then described by the SIFT descriptor (Lowe, 2004) and quantized by k-means to build the visual vocabulary of size 5000 . Each image includes about 1000 overlapping documents (squared shaped regions of the image), at different positions and scales. The images were split between training and testing according to the dataset specifications.

Comparison with baseline methods First, we would like to see how using multiple overlapping documents per image is better than a more trivial model. For comparison purposes we therefore developed 2 baseline methods.

(a) A patch based method: On training images the segmentation masks are used to fix the topic affectation and then estimate the probability for each topic to generate a particular word. We use $P(t \mid w)=\frac{P(w \mid t) P(t)}{P(w)}$ to compute the probability for an observed word to

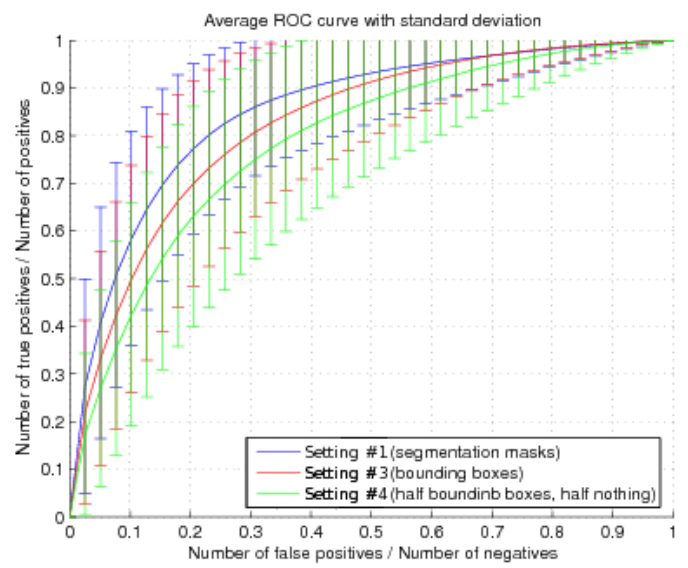

Figure 5: Average ROC curve obtained on all testing images, with the standard deviation (error bars).

belong to the foreground. Pixel level topic estimation is done in the same way as described in subsection 2.3.

(b) Single document method: The whole image constitute the only document in our model (as usually done in traditional LDA). Except for the mixture of documents, the rest of the method is the same.

The comparison is performed in a highly supervised framework: for all training images each patch label is known from the ground-truth (segmentation masks). In Figure 4, for different images the ROC curves are shown for the 3 methods together with the probability maps for the proposed methods and for one of the 2 baseline methods: the one with one document. The other baseline map is similar or slightly worse. These results typically illustrate the gain obtained using multiple overlapping documents in the image.

Influence of the supervision It is interesting to understand how much supervision is necessary to obtain good segmentation. Influence of supervision on segmentation accuracy is evaluated in the next series of experiments. Different kinds and levels of supervision were used: (a) segmentation masks which allow to give precisely which image patches belongs to objects, (b) bounding boxes alone, i.e. rectangle re-

\begin{tabular}{|l|l|l|}
\hline setting \#1 & all seg. mask & 0.798 \\
setting \#2 & $50 \%$ seg. masks, 50\% b.b. & 0.792 \\
setting \#3 & all b.b. (bounding boxes) & 0.761 \\
setting \#4 & $50 \%$ b.b., 50\% no info & 0.723 \\
\hline
\end{tabular}

Table 1: For each different supervision framework, its description and the mean EER obtained on the different ROC curves of pixel level classification 


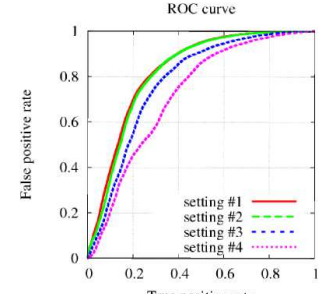

True positive rate
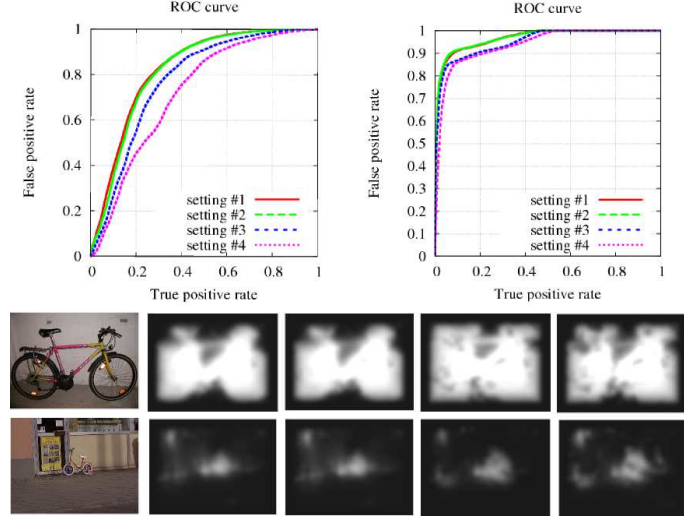

True positive ate

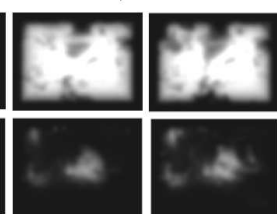

Figure 6: ROC curve and associated probability maps corresponding to different kinds of supervision.

gions of the image containing objects, (c) image labels (we only know the object is somewhere in the image, without knowing where), combined in 4 different settings, according to Table 1 . The question is to know how much the quality of the results depends on supervision.

For each of these supervision settings we produce probability maps for the tests images. Each probability map is used to compute a ROC curve and associated to EER. The EER average on all test images is displayed in the last column of Table 1 for the different settings.

We observed that the more supervision the more accurate the segmentation is, as expected. However, Table 1 shows that only a few masks are enough to ensure a stable estimation of image topics and satisfying segmentation. Even bounding boxes alone give reasonable results, even if the loss of accuracy is noticeable. As an illustration, Figure 6 shows the masks for the different settings described in Table 1.

Binary Masks At last, we show in Figure 7 binary segmentation masks obtained by thresholding probability maps. We can see the accuracy of the method, considering that no strong cues (color, texture, shape, etc.) are here to give evidence for pixels to belong to objects of interest.

\section{Conclusions and future works}

We have proposed a new method for learning to segment objects in images. Inspired by the LDA model, our model considers that images are made of multiple overlapping regions, treated as distinct documents. It increases the chance for small objects to be discov-

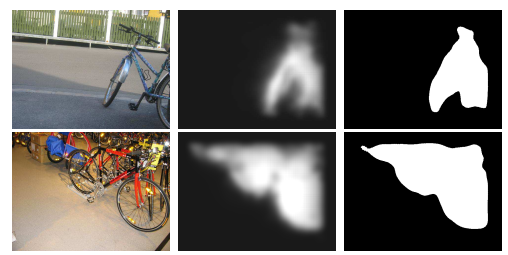

Figure 7: Several bike images of the Graz02 dataset (left), the probability map (middle) generated by our method and the segmentation mask (right) obtained using a threshold fixed for the database.

ered as being the main topic of at least one image sub-region. Used in a semi-supervised framework, it can achieve a remarkably high precision with difficult images even with relaxing supervision constraints.

However, further improvements could possibly make the performances even better. One of these improvements would be to embed local shape information within the model, making the detection of object boundaries more accurate.

\section{REFERENCES}

Blei, D., Ng, A., and Jordan, M. (2002). Latent dirichlet allocation. NIPS.

Borenstein, E., Sharon, E., and Ullman, S. (2004). Combining top-down and bottom-up segmentation. In $C V P R$.

Csurka, G., Dance, C., Fan, L., Williamowski, J., and Bray, C. (2004). Visual categorization with bags of keypoints. In ECCV workshop on Statistical Learning in Computer Vision.

Griffiths, T. and Steyvers, M. (2004). Finding scientific topics. In PNAS.

Haralick, R. and Shapiro, L. (1985). Image segmentation techniques. CVGIP.

Hofmann, T. (2001). Unsupervised learning by probabilistic latent semantic analysis. $M L$.

Kumar, M. P., Torr, P. H. S., and Zisserman, A. (2005). OBJ CUT. In $C V P R$.

Kumar, S. and Hebert, M. (2006). Discriminative random fields. IJCV.

Leibe, B. and Schiele, B. (2003). Interleaved object categorization and segmentation. In $B M V C$.

Lowe, D. G. (2004). Distinctive image features from scaleinvariant keypoints. IJCV.

Quattoni, A., Collins, M., and Darrell, T. (2004). Conditional random fields for object recognition. In NIPS.

Sivic, J., Russell, B., Efros, A., Zisserman, A., and Freeman, B. (2005). Discovering objects and their location in images. In ICCV.

Winn, J. and Shotton, J. (2006). The layout consistent random field for recognizing and segmenting partially occluded objects. In CVPR. 